\title{
Fuzzy semi pre-irresolute mappings in fuzzy topological spaces
}

\author{
M. Pritha*
}

\begin{abstract}
Y. C. Kim introduced $r$-fuzzy semi preopen ( $r$-fuzzy semi preclosed) sets in Ŝostak's fuzzy topological space. We investigate some properties of them. Moreover, we introduce and study fuzzy semi pre-irresolute mapping and fuzzy semi pre-connectedness in Ŝstak's fuzzy topological space.
\end{abstract}

\section{Keywords} $r$-fuzzy semi preopen (preclosed) sets, fuzzy semi pre-irresolute (semi pre-continuous) mapping, $r$-fuzzy semi pre-connected.

\section{AMS Subject Classification} 54A05, 54A10, 54A40.

Department of Mathematics, D.B. Jain College, Thoraipakkam, Chennai-600097, Tamil Nadu, India.

*Corresponding author: ${ }^{1}$ prithasrinivasan@gmail.com;

Article History: Received 28 November 2018; Accepted 09 April 2019

(C)2019 MJM

\section{Contents}

1 Introduction and preliminaries................296

$2 r$-fuzzy semi preopen and $r$-fuzzy semi preclosed sets 297

3 Fuzzy semi pre-irresolute mappings...........298

4 Fuzzy semi pre-connectedness.................300

References .............................. 301

\section{Introduction and preliminaries}

Sostak [7] introduced the fuzzy topology as an extension of Chang's fuzzy topology [1]. J. H. Park and B. Y. Lee [5] introduced fuzzy semi preopen and fuzzy semi preclosed sets in fuzzy topology.

In this paper, Y. C. Kim [4] introduced $r$-fuzzy semi preopen ( $r$-fuzzy semi preclosed) sets in Sostak's fuzzy topological space. Moreover, we establish some properties of fuzzy semi pre-continuous, fuzzy semi preopen (semi preclosed) maps and fuzzy semi pre-irresolute maps. Also, we investigate the relation between fuzzy semi pre-continuous maps and fuzzy semi pre irresolute maps. As a natural follow-up of the study of $r$-fuzzy semi preopen sets, we introduce and study $r$-fuzzy semi pre-connectedness of a fuzzy set.

Throughout this paper, let $X$ be a nonempty set, $I=[0,1]$ and $I_{0}=(0,1]$. For $\alpha \in I, \bar{\alpha}(x)=\alpha$ for all $x \in X$. The family of all fuzzy sets on $X$ denoted by $I^{X}$. For $\lambda, \mu \in I^{X}, \lambda$ is called quasi-coincident with $\mu$, denoted by $\lambda q \mu$, if there exists $x \in X$ such that $\lambda(x)+\mu(x)>1$. Otherwise we denote $\lambda \bar{q} \mu$.

Definition 1.1. [7] A function $\tau: I^{X} \rightarrow I$ is called a fuzzy topology on $X$ if it satisfies the following conditions:

$(O 1) \tau(\overline{0})=\tau(\overline{1})=1$,

(O2) $\tau\left(\bigvee_{i \in \Gamma} \mu_{i}\right) \geq \bigwedge_{i \in \Gamma} \tau\left(\mu_{i}\right)$, for any $\{\mu\}_{i \in \Gamma} \subset I^{X}$.

(O3) $\tau\left(\mu_{1} \wedge \mu_{2}\right) \geq \tau\left(\mu_{1}\right) \wedge \tau\left(\mu_{2}\right)$, for any $\mu_{1}, \mu_{2} \in I^{X}$.

The pair $(X, \tau)$ is called a fuzzy topological space (for short, fts).

Remark 1.2. [4] Let $(X, \tau)$ be a fuzzy topological space. Then, for each $r \in I, \tau_{r}=\left\{\mu \in I^{X}: \tau(\mu) \geq r\right\}$ is a Change's fuzzy topology on $X$.

Theorem 1.3. [2], [3] Let $(X, \tau)$ be a fts. Then for each $r \in I_{0}, \lambda \in I^{X}$ we define an operator $C_{\tau}: I^{X} \times I_{0} \rightarrow I^{X}$ as follows

$$
C_{\tau}(\lambda, r)=\bigwedge\left\{\mu \in I^{X}: \lambda \leq \mu, \tau(\overline{1}-\mu) \geq r\right\} .
$$

For $\lambda, \mu \in I^{X}$ and $r, s \in I_{0}$, the operator $C_{\tau}$ satisfies the following conditions:

(1) $C_{\tau}(\overline{0}, r)=\overline{0}$.

(2) $\lambda \leq C_{\tau}(\lambda, r)$

(3) $C_{\tau}(\lambda, r) \vee C_{\tau}(\mu, r)=C_{\tau}(\lambda \vee \mu, r)$.

(4) $C_{\tau}(\lambda, r) \leq C_{\tau}(\lambda, s)$ if $r \leq s$. 
(5) $C_{\tau}\left(C_{\tau}(\lambda, r), r\right)=C_{\tau}(\lambda, r)$.

Theorem 1.4. [3] Let $(X, \tau)$ be a fts. Then for each $r \in$ $I_{0}, \lambda \in I^{X}$ we define an operator $I_{\tau}: I^{X} \times I_{0} \rightarrow I^{X}$ as follows $I_{\tau}(\lambda, r)=\bigvee\left\{\mu \in I^{X}: \lambda \geq \mu, \tau(\mu) \geq r\right\}$.

For $\lambda, \mu \in I^{X}$ and $r, s \in I_{0}$, the operator $I_{\tau}$ satisfies the following conditions:

(1) $I_{\tau}(\overline{1}-\lambda, r)=\overline{1}-C_{\tau}(\lambda, r)$.

(2) $I_{\tau}(\overline{1}, r)=\overline{1}$.

(3) $\lambda \geq I_{\tau}(\lambda, r)$

(4) $I_{\tau}(\lambda, r) \wedge I_{\tau}(\mu, r)=I_{\tau}(\lambda \wedge \mu, r)$.

(5) $I_{\tau}(\lambda, r) \geq I_{\tau}(\lambda, s)$ if $r \leq s$.

(6) $I_{\tau}\left(I_{\tau}(\lambda, r), r\right)=I_{\tau}(\lambda, r)$.

(7) If $I_{\tau}\left(C_{\tau}(\lambda, r), r\right)=\lambda$, then $C_{\tau}\left(I_{\tau}(\overline{1}-\lambda, r), r\right)=\overline{1}-\lambda$.

Definition 1.5. [6] Let $(X, \tau)$ be a fts. For $\lambda \in I^{X}$ and $r \in I_{0}$.

(1) $\lambda$ is called $r$-fuzzy preopen ( $r$-fpo, for short) iff $\lambda \leq$ $I_{\tau}\left(C_{\tau}(\lambda, r), r\right)$.

(2) $\lambda$ is called $r$-fuzzy preclosed ( $r$-fpc, for short) iff $\overline{1}-\lambda$ is $r$-fuzzy preopen set of $X$.

(3) The $r$-fuzzy pre-interiror of $\lambda$, denoted by $P I_{\tau}(\lambda, r)$, is defined by $P I_{\tau}(\lambda, r)=\bigvee\left\{v \in I^{X}: v \leq \lambda, v\right.$ is $r$-fpo $\}$

(4) The $r$-fuzzy pre-closure of $\lambda$, denoted by $P C_{\tau}(\lambda, r)$, is defined by $P C_{\tau}(\lambda, r)=\bigwedge\left\{v \in I^{X}: v \geq \lambda, v\right.$ is $r$-fpc $\}$.

Definition 1.6. [4] Let $(X, \tau)$ be a fts. For $\lambda \in I^{X}$ and $r \in I_{0}$,

(1) $\lambda$ is called $r$-fuzzy semi preopen (for short, $r$-fspo) if $\lambda \leq C_{\tau}\left(I_{\tau}\left(C_{\tau}(\lambda, r), r\right), r\right)$

(2) $\lambda$ is called $r$-fuzzy semi preclosed (for short $r$-fspc) if $I_{\tau}\left(C_{\tau}\left(I_{\tau}(\lambda, r), r\right), r\right) \leq \lambda$.

Theorem 1.7. [4] Let $(X, \tau)$ be a fts. For $\lambda \in I^{X}$ and $r, s, r_{i} \in I_{0}$.

(1) $\lambda$ is $r$-fspo iff $\overline{1}-\lambda$ is $r$-fspc.

(2) Any union of $r$-fspo sets is $r$-fspo.

(3) Any intersection of $r$-fspc sets is $r$-fspc.

(4) If $\lambda$ is $r$-fpo, then $\lambda$ is $r$-fspo.

Definition 1.8. [3], [7] Let $(X, \tau)$ and $(Y, \eta)$ be a fts's. Let $f: X \rightarrow Y$ be a mapping.

(1) $f$ is called fuzzy continuous iff $\eta(\mu) \leq \tau\left(f^{-1}(\mu)\right)$ for each $\mu \in I^{Y}$.

(2) $f$ is called fuzzy open iff $\tau(\lambda) \leq \eta(f(\lambda))$ for each $\lambda \in I^{X}$.

(3) $f$ is called fuzzy closed iff $\tau(\overline{1}-\lambda) \leq \eta(\overline{1}-f(\lambda))$ for each $\lambda \in I^{X}$.

\section{2. $r$-fuzzy semi preopen and $r$-fuzzy semi preclosed sets}

Theorem 2.1. Let $(X, \tau)$ be a fts. For each $r \in I_{0}, \lambda \in I^{X}$, we define an operator $S P C_{\tau}: I^{X} \times I_{0} \rightarrow I^{X}$ as follows:

$\operatorname{SPC}_{\tau}(\lambda, r)=\bigwedge\left\{\mu \in I^{X}: \lambda \leq \mu, \mu\right.$ is $r$-fspc $\}$.

For $\lambda, \mu \in I^{X}$ and $r \in I_{0}$, it holds the following properties.

(1) $\operatorname{SPC}_{\tau}(\overline{0}, r)=\overline{0}$.

(2) $\lambda \leq S P C_{\tau}(\lambda, r)$.

(3) $\operatorname{SPC}_{\tau}(\lambda, r) \vee S P C_{\tau}(\mu, r) \leq S P C_{\tau}(\lambda \vee \mu, r)$.

(4) $\operatorname{SPC}_{\tau}\left(\operatorname{SPC}_{\tau}(\lambda, r), r\right)=\operatorname{SPC}_{\tau}(\lambda, r)$.

(5) $\operatorname{SPC}_{\tau}(\lambda, r)=\lambda$ iff $\lambda$ is $r$-fspc.

(6) $C_{\tau}\left(\operatorname{SPC}_{\tau}(\lambda, r), r\right)=S P C_{\tau}\left(C_{\tau}(\lambda, r), r\right)=C_{\tau}(\lambda, r)$.

(7) $\lambda$ is $r$-fspc iff $S P C_{\tau}(\lambda, r)=\lambda$.

Proof. (1), (2), (5), (7) are easily proved from the definition of $S P C_{\tau}$.

(3) Since $\lambda, \mu \leq \lambda \vee \mu$, we have

$$
S P C_{\tau}(\lambda, r) \vee S P C_{\tau}(\mu, r) \leq S P C_{\tau}(\lambda \vee \mu, r) .
$$

(4) From (2), we only show $\operatorname{SPC}_{\tau}(\lambda, r) \geq \operatorname{SPC}_{\tau}\left(P C_{\tau}(\lambda, r), r\right)$. Suppose

$$
\operatorname{SPC}_{\tau}(\lambda, r) \nsupseteq S P C_{\tau}\left(\operatorname{SPC}_{\tau}(\lambda, r), r\right) .
$$

There exist $x \in X$ and $t \in(0,1)$ such that

$$
\operatorname{SPC}_{\tau}(\lambda, r)(x)<t<\operatorname{SPC}_{\tau}\left(\operatorname{SPC}_{\tau}(\lambda, r), r\right)(x) .
$$

Since $\operatorname{SPC}_{\tau}(\lambda, r)(x)<t$, by the Definition of $S P C_{\tau}$, there exists $r$-fspc set $\lambda_{1}$ with $\lambda \leq \lambda_{1}$ such that

$$
\operatorname{SPC}_{\tau}(\lambda, r)(x) \leq \lambda_{1}(x)<t .
$$

Since $\lambda \leq \lambda_{1}$, we have $\operatorname{SPC}_{\tau}(\lambda, r) \leq \lambda_{1}$. Again, by the Definition of $S P C_{\tau}$,

$$
\operatorname{SPC}_{\tau}\left(\operatorname{SPC}_{\tau}(\lambda, r), r\right) \leq \lambda_{1}
$$

Hence $S P C_{\tau}\left(S P C_{\tau}(\lambda, r), r\right)(x) \leq \lambda_{1}(x)<t$. It is a contradiction for (A). Thus,

$\operatorname{SPC}_{\tau}(\lambda, r) \geq \operatorname{SPC}_{\tau}\left(\operatorname{SPC}_{\tau}(\lambda, r), r\right)$.

(6) From (2) and $C_{\tau}(\lambda, r)$ is $r$-fspc we have, $S P C_{\tau}\left(C_{\tau}(\lambda, r), r\right)=$ $C_{\tau}(\lambda, r)$. We only show that

$$
C_{\tau}\left(\operatorname{SPC}_{\tau}(\lambda, r), r\right)=C_{\tau}(\lambda, r) .
$$


Since $\lambda \leq \operatorname{SPC}_{\tau}(\lambda, r)$,

$$
C_{\tau}\left(\operatorname{SPC}_{\tau}(\lambda, r), r\right) \geq C_{\tau}(\lambda, r) .
$$

Suppose that

$$
C_{\tau}\left(\operatorname{SPC}_{\tau}(\lambda, r), r\right) \not \leq C_{\tau}(\lambda, r) .
$$

There exist $x \in X$ and $r \in I_{0}$ such that

$$
C_{\tau}\left(S P C_{\tau}(\lambda, r), r\right)(x)>C_{\tau}(\lambda, r)(x) .
$$

By the definition of $C_{\tau}$, there exists $\rho \in I^{X}$ with $\lambda \leq \rho$ and $\tau(\overline{1}-\rho) \geq r$ such that

$$
C_{\tau}\left(S P C_{\tau}(\lambda, r), r\right)(x)>\rho(x) \geq C_{\tau}(\lambda, r)(x) .
$$

On the other hand, since $\rho=C_{\tau}(\rho, r), \lambda \leq \rho$ implies

$C_{\tau}(\rho, r)=\rho$.

$$
S P C_{\tau}(\lambda, r) \leq S P C_{\tau}(\rho, r)=S P C_{\tau}\left(C_{\tau}(\rho, r), r\right)=
$$

Thus,

$$
C_{\tau}\left(\operatorname{SPC}_{\tau}(\lambda, r), r\right) \leq \rho .
$$

It is a contradiction. Hence $C_{\tau}\left(\operatorname{SPC}_{\tau}(\lambda, r), r\right) \leq C_{\tau}(\lambda, r)$.

Theorem 2.2. Let $(X, \tau)$ be a fts. Define an operator $S P I_{\tau}$ : $I^{X} \times I_{0} \rightarrow I^{X}$ as follows:

Then:

$\operatorname{SPI}_{\tau}(\lambda, r)=\bigvee\left\{\mu \in I^{X} \mid \mu \leq \lambda, \mu\right.$ is $r$-fspo $\}$.

(1) $S P I_{\tau}(\overline{1}-\lambda, r)=\overline{1}-S P C_{\tau}(\lambda, r)$.

(2) $I_{\tau}(\lambda, r) \leq P I_{\tau}(\lambda, r) \leq S P I_{\tau}(\lambda, r) \leq \lambda \leq S P C_{\tau}(\lambda, r) \leq$ $P C_{\tau}(\lambda, r) \leq C_{\tau}(\lambda, r)$.

(3) $\lambda$ is $r$-fspo iff $\operatorname{SPI}_{\tau}(\lambda, r)=\lambda$.

Proof. (1) For each $\lambda \in I^{X}$ and $r \in I_{0}$, we have $S P I_{\tau}(\overline{1}-\lambda, r)=\bigvee\left\{\mu \in I^{X} \mid \mu \leq \overline{1}-\lambda, \mu\right.$ is $r$-fspo $\}$ $r$-fspc $\}$

$$
=\overline{1}-\bigwedge\left\{\overline{1}-\mu \in I^{X} \mid \overline{1}-\mu \geq \lambda, \overline{1}-\mu\right. \text { is }
$$

(2) and (3) are easily proved.

$$
=\overline{1}-S P C_{\tau}(\lambda, r) \text {. }
$$

\section{Fuzzy semi pre-irresolute mappings}

Definition 3.1. Let $(X, \tau)$ and $(Y, \eta)$ be a fts's. Let $f: X \rightarrow$ $Y$ be a mapping.

(1) $f$ is fuzzy semi pre-irresolute (resp. fuzzy semi precontinuous [4]) iff $f^{-1}(\mu)$ is $r$-fspo set of $X$ for each $r$-fspo set $\mu \in I^{Y}$ (resp. $\eta(\mu) \geq r$ ).

(2) $f$ is fuzzy semi pre-irresolute open (resp. fuzzy semi pre-open [4]) iff $f(\mu)$ is $r$-fspo set of $Y$ for each $r$-fspo set $\mu \in I^{X}$ (resp. $\tau(\mu) \geq r$ ).
(3) $f$ is fuzzy semi pre-irresolute closed (resp. fuzzy semi pre-closed [4]) iff $f(\mu)$ is $r$-fspc set of $Y$ for each $r$-fspc set $\mu \in I^{X}$ (resp. $\tau(\overline{1}-\mu) \geq r$ ).

(4) $f$ is fuzzy semi pre-irresolute homeomorphism iff $f$ is bijective and both of $f$ and $f^{-1}$ are semi pre-irresolute.

Theorem 3.2. Let $\left(X, \tau_{1}\right)$ and $\left(Y, \tau_{2}\right)$ be a fts's. Let $f: X \rightarrow$ $Y$ be a mapping. The following statements are equivalent.

(1) A map $f$ is fuzzy semi pre-irresolute.

(2) For each $r$-fspc $\mu \in I^{Y}, f^{-1}(\mu)$ is $r$-fspc.

(3) $f\left(S P C_{\tau_{1}}(\lambda, r)\right) \leq S P C_{\tau_{2}}(f(\lambda), r)$, for each $\lambda \in I^{X}$ and $r \in I_{0}$.

(4) $\operatorname{SPC}_{\tau_{1}}\left(f^{-1}(\mu), r\right) \leq f^{-1}\left(\operatorname{SPC}_{\tau_{2}}(\mu, r)\right)$, for each $\mu \in I^{Y}$ and $r \in I_{0}$.

(5) $f^{-1}\left(S P I_{\tau_{2}}(\mu, r)\right) \leq S P I_{\tau_{1}}\left(f^{-1}(\mu), r\right)$, for each $\mu \in I^{Y}$ and $r \in I_{0}$.

Proof. $(1) \Leftrightarrow(2)$ It is easily proved from Theorem 1.7 , and $f^{-1}(\overline{1}-\mu)=\overline{1}-f^{-1}(\mu)$.

(2) $\Rightarrow$ (3) Suppose there exist $\lambda \in I^{X}$ and $r \in I_{0}$ such that

$$
f\left(S P C_{\tau_{1}}(\lambda, r)\right) \not \leq S P C_{\tau_{2}}(f(\lambda), r) .
$$

There exist $y \in Y$ and $t \in I_{0}$ such that

$$
f\left(S P C_{\tau_{1}}(\lambda, r)\right)(y)>t>S P C_{\tau_{2}}(f(\lambda), r)(y) .
$$

If $f^{-1}(\{y\})=\phi$, it is a contradiction because $f\left(\operatorname{SPC}_{\tau_{1}}(\lambda, r)\right)(y)=$ 0 .

If $f^{-1}(\{y\}) \neq \phi$, there exists $x \in f^{-1}(\{y\})$ such that (A)

$f\left(S P C_{\tau_{1}}(\lambda, r)\right)(y) \geq S P C_{\tau_{1}}(\lambda, r)(x)>t>S P C_{\tau_{2}}(f(\lambda), r)(f(x))$.

Since $S P C_{\tau_{2}}(f(\lambda), r)(f(x))<t$, there exists $r$-fspc $\mu \in I^{Y}$ with $f(\lambda) \leq \mu$ such that

$$
\operatorname{SPC}_{\tau_{2}}(f(\lambda), r)(f(x)) \leq \mu(f(x))<t .
$$

Moreover, $f(\lambda) \leq \mu$ implies $\lambda \leq f^{-1}(\mu)$. From (2), $f^{-1}(\mu)$ is $r$-fspc. Thus, $S P C_{\tau_{1}}(\lambda, r)(x) \leq f^{-1}(\mu)(x)=\mu(f(x))<t$. It is a contradiction for (A).

(3) $\Rightarrow$ (4) For all $\mu \in I^{Y}, r \in I_{0}$, put $\lambda=f^{-1}(\mu)$. From (3), we have

$$
f\left(S P C_{\tau_{1}}\left(f^{-1}(\mu), r\right)\right) \leq S P C_{\tau_{2}}\left(f\left(f^{-1}(\mu)\right), r\right) \leq S P C_{\tau_{2}}(\mu, r) .
$$

It implies

$$
\left.S P C_{\tau_{1}}\left(f^{-1}(\mu), r\right)\right) \leq f^{-1}\left(f\left(S P C_{\tau_{1}}\left(f^{-1}(\mu), r\right)\right)\right) \leq f^{-1}\left(S P C_{\tau_{2}}(\mu, r)\right) .
$$


$(4) \Rightarrow(5)$ It is easily proved from Theorem 3(1)

(5) $\Rightarrow(1)$ Let $\mu$ be $r$-fspo set of $Y$. From Theorem 3(3), $\mu=S P I_{\tau_{2}}(\mu, r)$. By (5),

$$
f^{-1}(\mu) \leq S P I_{\tau_{1}}\left(f^{-1}(\mu), r\right) .
$$

On the other hand, by Theorem 3(2),

$$
f^{-1}(\mu) \geq S P I_{\tau_{1}}\left(f^{-1}(\mu), r\right)
$$

Thus, $f^{-1}(\mu)=S P I_{\tau_{1}}\left(f^{-1}(\mu), r\right)$, that is, $f^{-1}(\mu)$ is $r$-fspo.

The following theorem is similarly proved as Theorem 3.2

Theorem 3.3. Let $\left(X, \tau_{1}\right)$ and $\left(Y, \tau_{2}\right)$ be a fts's. Let $f: X \rightarrow$ $Y$ be a mapping. The following statements are equivalent.

(1) A map $f$ is fuzzy semi pre-continuous.

(2) $f\left(S P C_{\tau_{1}}(\lambda, r)\right) \leq C_{\tau_{2}}(f(\lambda), r)$, for each $\lambda \in I^{X}$ and $r \in$ $I_{0}$.

(3) $S P C_{\tau_{1}}\left(f^{-1}(\mu), r\right) \leq f^{-1}\left(C_{\tau_{2}}(\mu, r)\right)$, for each $\mu \in I^{Y}$ and $r \in I_{0}$.

(4) $f^{-1}\left(I_{\tau_{2}}(\mu, r)\right) \leq S P I_{\tau_{1}}\left(f^{-1}(\mu), r\right)$, for each $\mu \in I^{Y}$ and $r \in I_{0}$.

Theorem 3.4. Let $(X, \tau)$ and $(Y, \eta)$ be a fts's. Let $f$ : $X \rightarrow Y$ be a bijective mapping. The following statements are equivalent.

(1) A map $f$ is fuzzy semi pre-irresolute.

(2) $\operatorname{SPI}_{\eta}(f(\lambda), r) \leq f\left(\operatorname{SPI}_{\tau}(\lambda, r)\right)$, for each $\lambda \in I^{X}$ and $r \in I_{0}$.

Proof. $(1) \Rightarrow(2)$ : Let $f$ be fuzzy semi pre-irresolute mapping and $\lambda \in I^{X}, r \in I_{0}$. Then $f^{-1}\left(\operatorname{SPI}_{\eta}(f(\lambda), r)\right)$ is $r$-fspo set in $X$. By Theorem 3.2 and the fact that $f$ is one-to-one we have

$$
\begin{gathered}
f^{-1}\left(\operatorname{SPI}_{\eta}(f(\lambda), r)\right) \leq S P I_{\tau}\left(f^{-1}(f(\lambda), r)\right) \\
=S P I_{\tau}(\lambda, r) .
\end{gathered}
$$

Again since $f$ is onto we have

$$
\begin{gathered}
\left.S P I_{\eta}(f(\lambda), r)\right)=f f^{-1}\left(S P I_{\eta}(f(\lambda), r)\right) \\
\leq f\left(S P I_{\tau}(\lambda, r)\right) .
\end{gathered}
$$

(2) $\Rightarrow(1)$ : Let $\mu$ be $r$-fspo set of $Y$. Then by Theorem 3(3), $\mu=S P I_{\eta}(\mu, r)$. By (2)

$$
\begin{gathered}
f\left(\operatorname{SPI}_{\tau}\left(f^{-1}(\mu), r\right)\right) \geq \operatorname{SPI}_{\eta}\left(f f^{-1}(\mu), r\right) \\
\left.=\operatorname{SPI}_{\eta}(\mu, r)\right) \\
=\mu .
\end{gathered}
$$

It implies

$$
\begin{gathered}
\left.S P I_{\tau}\left(f^{-1}(\mu), r\right)\right)=f^{-1} f\left(S P I_{\tau}\left(f^{-1}(\mu), r\right)\right. \\
\geq f^{-1}(\mu) .
\end{gathered}
$$

Hence, $f^{-1}(\mu)=S P I_{\tau}\left(f^{-1}(\mu), r\right)$, that is, $f^{-1}(\mu)$ is $r$-fspo set of $X$.

Theorem 3.5. Let $(X, \tau)$ and $(Y, \eta)$ be fts's. Let $f: X \rightarrow Y$ be a mapping. The following statements are equivalent:

(1) $f$ is called fuzzy semi-pre-irresolute open.

(2) $f\left(S P I_{\tau}(\lambda, r)\right) \leq S P I_{\eta}(f(\lambda), r)$, for each $\lambda \in I^{X}$ and $r \in I_{0}$.

(3) $\operatorname{SPI}_{\tau}\left(f^{-1}(\mu), r\right) \leq f^{-1}\left(\operatorname{SPI}_{\eta}(\mu, r)\right)$, for each $\mu \in I^{Y}$ and $r \in I_{0}$.

(4) For any $\mu \in I^{Y}$ and any $r$-fpsc $\lambda \in I^{X}$ with $f^{-1}(\mu) \leq$ $\lambda$, there exits a $r$-fspc $\rho \in I^{Y}$ with $\mu \leq \rho$ such that $f^{-1}(\rho) \leq \lambda$.

Proof. $(1) \Rightarrow(2)$ : For each $\lambda \in I^{X}$, since $S P I_{\tau}(\lambda, r) \leq \lambda$ from Theorem (2), we have $f\left(S P I_{\tau}(\lambda, r)\right) \leq f(\lambda)$. From (1), $f\left(S P I_{\tau}(\lambda, r)\right)$ is $r$-fspo. Hence $f\left(S P I_{\tau}(\lambda, r)\right) \leq S P I_{\eta}(f(\lambda), r)$.

(2) $\Rightarrow$ (3): For all $\mu \in I^{Y}, r \in I_{0}$, put $\lambda=f^{-1}(\mu)$ from (2). Then $\quad f\left(S P I_{\tau}\left(f^{-1}(\mu), r\right) \leq S P I_{\eta}\left(f\left(f^{-1}(\mu)\right), r\right) \leq\right.$ $S P I_{\eta}(\mu, r)$.

It implies $S P I_{\tau}\left(f^{-1}(\mu), r\right) \leq f^{-1}\left(S P I_{\eta}(\mu, r)\right)$.

(3) $\Rightarrow(4)$ : Let $\lambda$ be $r$-fspc set of $X$ such that $f^{-1}(\mu) \leq \lambda$. Since $\overline{1}-\lambda \leq f^{-1}(\overline{1}-\mu)$ and $\operatorname{SPI}_{\tau}(\overline{1}-\lambda, r)=\overline{1}-\lambda$,

$$
S P I_{\tau}(\overline{1}-\lambda, r)=\overline{1}-\lambda \leq S P I_{\tau}\left(f^{-1}(\overline{1}-\mu), r\right) \text {. }
$$

From(3),

$\mu, r))$.

$$
\overline{1}-\lambda \leq \operatorname{SPI}_{\tau}\left(f^{-1}(\overline{1}-\mu), r\right) \leq f^{-1}\left(\operatorname{SPI}_{\eta}(\overline{1}-\right.
$$

It implies

$$
\begin{aligned}
\lambda & \geq \overline{1}-f^{-1}\left(\operatorname{SPI}_{\eta}(\overline{1}-\mu, r)\right) \\
& =f^{-1}\left(\overline{1}-\operatorname{SPI}_{\eta}(\overline{1}-\mu, r)\right) \\
& =f^{-1}\left(\operatorname{SPC}_{\eta}(\mu, r)\right) .
\end{aligned}
$$

Hence there exists a $r$-fspc $\operatorname{SPC}_{\eta}(\mu, r) \in I^{Y}$ with $\mu \leq \operatorname{SPC}_{\eta}(\mu, r)$ such that $f^{-1}\left(\operatorname{SPC}_{\eta}(\mu, r)\right) \leq r \lambda$.

(4) $\Rightarrow(1)$ : Let $\omega$ be $r$-fspo set $X$. Put $\mu=\overline{1}-f(\omega)$ and $\lambda=\overline{1}-\omega$ such that $\lambda$ is $r$-fspc. We obtain

$\overline{1}-\omega=\lambda$

$$
f^{-1}(\mu)=f^{-1}(\overline{1}-f(\omega))=\overline{1}-f^{-1}(f(\omega)) \leq
$$

From(4), there exists $r$-fspc set $\rho$ with $\mu \leq \rho$ such that $f^{-1}(\rho) \leq$ $\lambda=\overline{1}-\omega$. It implies $\omega \leq \overline{1}-f^{-1}(\rho)=f^{-1}(\overline{1}-\rho)$. Thus, $f(\omega) \leq f\left(f^{-1}(\overline{1}-\rho)\right) \leq \overline{1}-\rho$. On the other hand, since $\mu \leq \rho$, 


$$
f(\omega)=\overline{1}-\mu \geq \overline{1}-\rho .
$$

Hence $f(\omega)=\overline{1}-\rho$, that is, $f(\omega)$ is $r$-fspo. 3.5

The following theorem is similarly proved from Theorem

Theorem 3.6. Let $(X, \tau)$ and $(Y, \eta)$ be a fts's. Let $f: X \rightarrow Y$ be a mapping. The following statements are equivalent:

(1) $f$ is called fuzzy semi pre-irresolute closed.

(2) $f\left(\operatorname{SPC}_{\tau}(\lambda, r)\right) \leq \operatorname{SPC}_{\eta}(f(\lambda), r)$, for each $\lambda \in I^{X}$ and $r \in I_{0}$.

Theorem 3.7. Let $(X, \tau)$ and $(Y, \eta)$ be a fts's. Let $f: X \rightarrow Y$ be a bijective mapping.

(1) $f$ is a fuzzy semi pre-irresolute closed map iff, for each $\mu \in I^{Y}$ and $r \in I_{0}, f^{-1}\left(\operatorname{SPC}_{\eta}(\mu, r)\right) \leq S P C_{\tau}\left(f^{-1}(\mu), r\right)$.

(1) $f$ is a fuzzy semi pre-irresolute closed map iff $f$ is a fuzzy semi pre-irresolute open map.

Proof. $(1)(\Rightarrow)$ Let $f$ be fuzzy semi pre-irresolute closed. From Theorem 3.6(2), for each $\lambda \in I^{X}$ and $r \in I_{0}$.

$$
f\left(\operatorname{SPC}_{\tau}(\lambda, r)\right) \geq \operatorname{SPC}_{\eta}(f(\lambda), r) .
$$

For all $\mu \in I^{Y}, r \in I_{0}$. Put $\lambda=f^{-1}(\mu)$ from (2) since $f$ is onto, $f\left(f^{-1}(\mu)\right)=\mu$. Thus,

$$
\begin{gathered}
f\left(\operatorname{SPC}_{\tau}\left(f^{-1}(\mu), r\right)\right) \geq \operatorname{SPC}_{\eta}\left(f\left(f^{-1}(\mu)\right), r\right) . \\
=\operatorname{SPC} C_{\eta}(\mu, r) .
\end{gathered}
$$

It implies

$$
\begin{gathered}
\left.S P C_{\tau}\left(f^{-1}(\mu), r\right)\right)=f^{-1}\left(f\left(\operatorname{SPC}_{\tau}\left(f^{-1}(\mu), r\right)\right)\right) \\
\geq f^{-1}\left(\operatorname{SPC}_{\eta}(\mu, r)\right) .
\end{gathered}
$$

$(\Leftarrow)$ Put $\mu=f(\lambda)$. Since $f$ is injective,

$$
\begin{gathered}
f^{-1}\left(S P C_{\eta}(f(\lambda), r)\right) \leq S P C_{\tau}\left(f^{-1}(f(\lambda)), r\right) \\
=S P C_{\tau}(\lambda, r) .
\end{gathered}
$$

Since $f$ is onto,

$$
\operatorname{SPC}_{\eta}(f(\lambda), r) \leq f\left(\operatorname{SPC}_{\tau}(\lambda, r)\right) .
$$

(2) It is easily proved from:

$$
\begin{array}{rlrl} 
& & f^{-1}\left(\operatorname{SPC}_{\eta}(\mu, r) \leq S P C_{\tau}\left(f^{-1}(\mu), r\right)\right. \\
\left.f^{-1}(\mu), r\right) & \Leftrightarrow \quad f^{-1}\left(\overline{1}-\operatorname{SPI}_{\eta}(\overline{1}-\mu, r)\right) \leq \overline{1}-\operatorname{SPI}_{\tau}(\overline{1}- \\
S P I_{\tau}\left(f^{-1}(\overline{1}-\mu), r\right) . & \Leftrightarrow \quad f^{-1}\left(\operatorname{SPI}_{\eta}(\overline{1}-\mu, r)\right) &
\end{array}
$$

From the above Theorems we obtain the following theorem.
Theorem 3.8. Let $f:(X, \tau) \rightarrow(Y, \eta)$ be a bijective mapping from a fts $(X, \tau)$ into a fts $(Y, \eta)$. For each $\lambda \in I^{X}, \mu \in I^{Y}$ and $r \in I_{0}$. The following statements are equivalent:

(1) $f$ is a fuzzy semi pre-irresolute homeomorphism.

(2) $f$ is a fuzzy semi pre-irresolute and fuzzy semi preirresolute open.

(3) $f$ is a fuzzy semi pre-irresolute and fuzzy semi preirresolute closed.

(4) $f\left(S P I_{\tau}(\lambda, r)\right)=S P I_{\eta}(f(\lambda), r)$.

(5) $f\left(\operatorname{SPC}_{\tau}(\lambda, r)\right)=\operatorname{SPC}_{\eta}(f(\lambda), r)$.

(6) $\operatorname{SPI}_{\tau}\left(f^{-1}(\mu), r\right)=f^{-1}\left(\operatorname{SPI}_{\eta}(\mu, r)\right)$.

(7) $\operatorname{SPC}_{\tau}\left(f^{-1}(\mu), r\right)=f^{-1}\left(\operatorname{SPC}_{\eta}(\mu, r)\right)$.

Remark 3.9. For the mapping $f: X \rightarrow Y$, the following statements are valid:

(1) $f$ is a fuzzy semi pre-irresolute $\Rightarrow f$ is a fuzzy semi pre-continuous.

(2) $f$ is a fuzzy continuous $\Rightarrow f$ is a fuzzy semi pre-continuous.

The converses of Remark 3.9(1,2) need not be true from the following example.

Example 3.10. Let $\lambda_{1}, \lambda_{2}, \lambda_{3}$ and $\lambda_{4}$ be fuzzy subsets of $X=\{a, b, c\}$ defined as follows:

$$
\begin{aligned}
& \lambda_{1}(a)=0.2, \lambda_{1}(b)=0.4, \lambda_{1}(c)=0.5, \\
& \lambda_{2}(a)=0.2, \lambda_{2}(b)=0.2, \lambda_{2}(c)=0.2, \\
& \lambda_{3}(a)=0.5, \lambda_{3}(b)=0.5, \lambda_{3}(c)=0.5, \\
& \lambda_{4}(a)=0.6, \lambda_{4}(b)=0.6, \lambda_{4}(c)=0.6 .
\end{aligned}
$$

Then $\tau, \eta: I^{X} \rightarrow I$ defined as

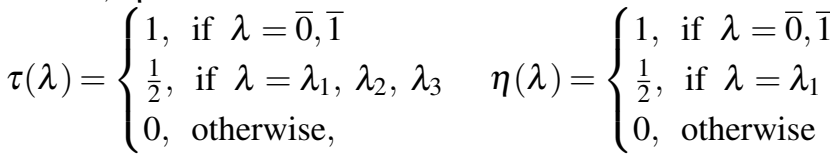
are fuzzy topologies on $X$. Then, the identity mapping $i d_{X}$ : $(X, \tau) \rightarrow(X, \eta)$ is fuzzy continuous and fuzzy semi pre continuous but not fuzzy semi pre irresolute, because $\lambda_{4}$ is $\frac{1}{2}$-fspo but $f^{-1}\left(\lambda_{4}\right)$ is not $\frac{1}{2}$-fspo from

$\lambda_{4} \leq C_{\tau}\left(I_{\tau}\left(C_{\tau}\left(\lambda_{5}, \frac{1}{2}\right), \frac{1}{2}\right), \frac{1}{2}\right)$

$\lambda_{5} \leq \overline{1}$

$\lambda_{4} \leq C_{\tau}\left(I_{\tau}\left(C_{\tau}\left(\lambda_{4}, \frac{1}{2}\right), \frac{1}{2}\right), \frac{1}{2}\right)$

$=C_{\tau}\left(I_{\tau}\left(\lambda_{2}^{c}, \frac{1}{2}\right), \frac{1}{2}\right)$

$=I_{\tau}\left(\lambda_{3}, \frac{1}{2}\right)$

$\not \leq \lambda_{3}$.

\section{Fuzzy semi pre-connectedness}

Definition 4.1. Let $(X, \tau)$ be a fts and $\lambda, \mu \in I^{X}, r \in I_{0}$. The two fuzzy sets $\lambda$ and $\mu$ are said to be $r$-fuzzy semi preseparated iff $\lambda \bar{q} S P C_{\tau}(\mu, r)$ and $\mu \bar{q} S P C_{\tau}(\lambda, r)$. 
Definition 4.2. A fuzzy set which cannot be expressed as the union of two $r$-fuzzy semi pre-separated sets is said to be $r$-fuzzy semi pre-connected set.

Theorem 4.3. Let $(X, \tau)$ be a fts and $\lambda, \mu \in I^{X}, r \in I_{0}$.

(i) If $\lambda, \mu$ are $r$-fuzzy semi-pre-separated and $v, \eta$ are non-null fuzzy sets such that $v \leq \lambda, \eta \leq \mu$, then $v, \eta$ are also $r$-fuzzy semi pre separated.

(ii) If $\lambda \bar{q} \mu$ and either both are $r$-fspo or both $r$-fspc, then $\lambda$ and $\mu$ are $r$-fuzzy semi pre separated.

(iii) If $\lambda, \mu$ are either both $r$-fspo or both $r$-fspc, then $\lambda \wedge$ $(\overline{1}-\mu)$ and $\mu \wedge(\overline{1}-\lambda)$ are $r$-fuzzy semi pre-separated.

Proof. (1) and (2) are obvious.

(3) Let $\lambda$ and $\mu$ be both $r$-fspo. Since $\lambda \wedge(\overline{1}-\mu) \leq$ $\overline{1}-\mu, \operatorname{SPC}_{\tau}(\lambda \wedge(\overline{1}-\mu), r) \leq \overline{1}-\mu$ and hence $\operatorname{SPC}_{\tau}(\lambda \wedge$ $(\overline{1}-\mu), r) \bar{q} \mu$. Then

$$
S P C_{\tau}(\lambda \wedge(\overline{1}-\mu), r) \bar{q}(\mu \wedge(\overline{1}-\lambda)) .
$$

Again, since $\mu \wedge(\overline{1}-\lambda) \leq \overline{1}-\lambda, S_{\tau} C_{\tau}(\mu \wedge(\overline{1}-\lambda), r) \leq$ $\overline{1}-\lambda$ and hence $S P C_{\tau}(\mu \wedge(\overline{1}-\lambda), r) \bar{q} \lambda$. Then

$$
\operatorname{SPC}_{\tau}(\mu \wedge(\overline{1}-\lambda), r) \bar{q}(\lambda \wedge(\overline{1}-\mu)) .
$$

Thus $\lambda \wedge(\overline{1}-\mu)$ and $\mu \wedge(\overline{1}-\lambda)$ are $r$-fuzzy semi-pre-separated. Similarly we can prove when $\lambda$ and $\mu$ are $r$-fspc.

Theorem 4.4. Let $(X, \tau)$ be a fts and $r \in I_{0}$. The two non-null fuzzy sets $\lambda$ and $\mu$ are $r$-fuzzy semi pre-separated iff there exists two $r$-fspo sets $v, \omega$ such that $\lambda \leq v, \mu \leq \omega, \lambda \bar{q} \omega$ and $\mu \bar{q} v$.

Proof. For two $r$-fuzzy semi pre-separated sets $\lambda$ and $\mu$, $\mu \leq \overline{1}-S P C_{\tau}(\lambda, r)=\omega$ (say) and $\lambda \leq \overline{1}-S P C_{\tau}(\mu, r)=v$ (say), where $\omega$ and $v$ are clearly $r$-fspo, then $\omega \bar{q} S P C_{\tau}(\lambda, r)$ and $v \bar{q} S P C_{\tau}(\mu, r)$. Thus, $\lambda \bar{q} \omega$ and $\mu \bar{q} v$.

(Conversely) Let $v$ and $\omega$ be $r$-fspo sets such that $\lambda \leq v$, $\mu \leq \omega, \lambda \bar{q} \omega$ and $\mu \bar{q} v$. Then $\lambda \leq \overline{1}-\omega, \mu \leq \overline{1}-v$. Hence $S P C_{\tau}(\lambda, r) \leq \overline{1}-\omega, S P C_{\tau}(\mu, r) \leq \overline{1}-v$, which in turn imply that $S P C_{\tau}(\lambda, r) \bar{q} \mu$ and $S P C_{\tau}(\mu, r) \bar{q} \lambda$. Thus $\lambda$ and $\mu$ are $r$ fuzzy semi-pre-separated.

Theorem 4.5. Let $(X, \tau)$ be a fts, $r \in I_{0}$ and $\lambda$ be a non-null $r$-fuzzy semi pre-connected set. If $\lambda \leq \mu \leq S P C_{\tau}(\lambda, r)$ then $\mu$ is also $r$-fuzzy semi pre-connected.

Proof. Suppose that $\mu$ is not $r$-fuzzy semi pre-connected. Then there exists $r$-fuzzy semi pre-separated sets $\omega_{1}$ and $\omega_{2}$ in $X$ such that $\mu=\omega_{1} \vee \omega_{2}$. Let $v=\lambda \wedge \omega_{1}$ and $\omega=\lambda \wedge \omega_{2}$. Then $\lambda=v \vee \omega$. Since $v \leq \omega_{1}$ and $\omega \leq \omega_{2}$, by Theorem 4.3(1), $v$ and $\omega$ are $r$-fuzzy semi pre-separated, contradicting the $r$-fuzzy semi pre-connectedness of $\lambda$. Thus $\mu$ is $r$-fuzzy semi pre-connected.

Theorem 4.6. Let $f: X \rightarrow Y$ be a fuzzy semi pre-irresolute mapping, $\lambda \in I^{X}$ and $r \in I_{0}$. If $\lambda$ is $r$-fuzzy semi pre-connected set in $X$, then so is $f(\lambda)$ in $Y$.
Proof. Suppose that $f(\lambda)$ is not $r$-fuzzy semi pre-connected in $Y$. Then there exists $r$-fuzzy semi pre-separated sets $\mu$ and $v$ in $Y$ such that $f(\lambda)=\mu \vee v$. Since $\mu$ and $v$ are $r$-fuzzy semi pre-separated, by Theorem 4.4 there exists two $r$-fspo sets $\omega_{1}$ and $\omega_{2}$ such that $\mu \leq \omega_{1}, v \leq \omega_{2}, \mu \bar{q} \omega_{2}$ and $v \bar{q} \omega_{1}$. Now, $f$ being semi pre-irresolute, $f^{-1}\left(\omega_{1}\right)$ and $f^{-1}\left(\omega_{2}\right)$ are $r$-fspo sets in $X$ and

$$
\lambda \leq f^{-1} f(\lambda)=f^{-1}(\mu \vee v)=f^{-1}(\mu) \vee f^{-1}(v)
$$

For $\mu \bar{q} \omega_{2}$ and $v \bar{q} \omega_{1}$, we have $\mu \leq \overline{1}-\omega_{2}$ and $v \leq \overline{1}-\omega_{1}$ i.e., $f^{-1}(\mu) \leq \overline{1}-f\left(\omega_{2}\right)$ and $f^{-1}(v) \leq \overline{1}-f\left(\omega_{1}\right)$. Hence $f^{-1}(\mu) \bar{q} f^{-1}\left(\omega_{2}\right)$ and $f^{-1}(v) \bar{q} f^{-1}\left(\omega_{1}\right)$. By Theorem 4.4, $f^{-1}(\mu)$ and $f^{-1}(v)$ are $r$-fuzzy semi pre-separated in $X$. Since $\lambda=\left(\lambda \wedge f^{-1}(\mu)\right) \vee\left(\lambda \wedge f^{-1}(v)\right)$ and $\lambda \wedge f^{-1}(\mu)$ and $\lambda \wedge$ $f^{-1}(v)$ are $r$-fuzzy semi pre-separated in $X$ from Theorem 4.3 (1), $\lambda$ is not $r$-fuzzy semi pre-connected set. It is a contradiction.

Corollary 4.7. Let $f: X \rightarrow Y$ be a fuzzy semi pre-irresolute mapping and $r \in I_{0}$. If $X$ is $r$-fuzzy semi pre-connected, then so is $f(X)$.

\section{References}

[1] C.L. Chang, Fuzzy topological spaces, J. Math. Anal. Appl., 24 (1968), 182-190.

[2] K.C. Chattopadyay and S.K. Samanta, Fuzzy topology: fuzzy closure, fuzzy compactness and fuzzy connectedness, Fuzzy Sets and Systems, 54 (1993), 207-212.

[3] U. Höhle and S.E. Rodabaugh, Logic, Topology and Measure Theorey, The Handbooks of Fuzzy Sets Series, Kluwer Academic Publishers, Boston (1999).

[4] Y.C. Kim, A.A. Ramadan and S.E. Abbas, Weaker forms of continuity in Ŝstak's fuzzy topology, Indian J. Pure and Appl. Math., (2003), 311-333.

[5] J.H. Park and B.Y. Lee, Fuzzy semi preopen sets and fuzzy semi pre continuous mappings, Fuzzy Sets and Systems, 67(3) (1994), 359-364.

[6] Seok Jong Lee and Eun Pyo Lee, Fuzzy $r$-preopen sets and fuzzy $r$-pre-continuous maps, Bull. Korean Math. Soc., 36(1) (1999), 91-108.

[7] A.P. Ŝstak, On a fuzzy topological structure, Rend. Circ. Matem. Palermo Ser. II, 11 (1985), 89-103. 